\title{
Blockade of the Complement C5a/C5aR1 Axis Impairs Lung Cancer Bone Metastasis by CXCL16-mediated Effects
}

\author{
Daniel Ajona ${ }^{1,2,3,4}$, Carolina Zandueta ${ }^{1,2,3}$, Leticia Corrales ${ }^{1}$, Haritz Moreno ${ }^{1,2}$, María J. Pajares ${ }^{1,2,3,5}$, \\ Sergio Ortiz-Espinosa ${ }^{1,3,4}$, Elena Martínez-Terroba ${ }^{1,5}$, Naiara Perurena ${ }^{1}$, Fernando J. de Miguel ${ }^{1,4}$, \\ Eloisa Jantus-Lewintre ${ }^{3,6,7}$, Carlos Camps ${ }^{3,6,8,9}$, Silvestre Vicent ${ }^{1,2,3,5}$, Jackeline Agorreta ${ }^{1,2,3,5}$, \\ Luis M. Montuenga ${ }^{1,2,3,5}$, Ruben Pio ${ }^{1,2,3,4 \star}$, and Fernando Lecanda ${ }^{1,2,3,5 \star}$
}

${ }^{1}$ Center for Applied Medical Research, Program in Solid Tumors and Biomarkers, Pamplona, Spain; ${ }^{2}$ IdiSNA (Navarra Institute for Health Research), Pamplona, Spain; ${ }^{3}$ CIBERONC (Centro de Investigación Biomédica en Red de Cáncer), Spain; ${ }^{4}$ Department of Biochemistry and Genetics, School of Sciences, and ${ }^{5}$ Department of Histology and Pathology, School of Medicine, University of Navarra, Pamplona, Spain; ${ }^{6}$ Molecular Oncology Laboratory, Fundación Investigación, Hospital General Universitario de Valencia, Valencia, Spain; ${ }^{7}$ Department of Biotechnology, Universitat Politècnica de València, Valencia, Spain; ' ${ }^{8}$ Department of Medical Oncology, Hospital General Universitario de Valencia, Valencia, Spain; and ${ }^{9}$ Department of Medicine, Universitat de València, Valencia, Spain

\begin{abstract}
Rationale: C5aR1 (CD88), a receptor for complement anaphylatoxin $\mathrm{C} 5 \mathrm{a}$, is a potent immune mediator. Its impact on malignant growth and dissemination of non-small cell lung cancer cells is poorly understood.

Objectives: To investigate the contribution of the $\mathrm{C} 5 \mathrm{a} / \mathrm{C} 5 \mathrm{aR} 1$ axis to the malignant phenotype of non-small cell lung cancer cells, particularly in skeletal colonization, a preferential lung metastasis site.
\end{abstract}

Methods: Association between $\mathrm{C} 5 \mathrm{aR} 1$ expression and clinical outcome was assessed in silico and validated by immunohistochemistry. Functional significance was evaluated by lentiviral gene silencing and ligand L-aptamer inhibition in in vivo models of lung cancer bone metastasis. In vitro functional assays for signaling, migration, invasion, metalloprotease activity, and osteoclastogenesis were also performed.

Measurements and Main Results: High levels of C5aR1 in human lung tumors were significantly associated with shorter recurrence-free survival, overall survival, and bone metastasis. Silencing of C5aR1 in lung cancer cells led to a substantial reduction in skeletal metastatic burden and osteolysis in in vivo models. Furthermore, metalloproteolytic, migratory, and invasive tumor cell activities were modulated in vitro by $\mathrm{C} 5 \mathrm{aR} 1$ stimulation or gene silencing. L-Aptamer blockade or C5aR1 silencing significantly reduced the osseous metastatic activity of lung cancer cells in vivo. This effect was associated with decreased osteoclastogenic activity in vitro and was rescued by the exogenous addition of the chemokine CXCL16.

Conclusions: Disruption of C5aR1 signaling in lung cancer cells abrogates their tumor-associated osteoclastogenic activity, impairing osseous colonization. This study unveils the role played by the $\mathrm{C} 5 \mathrm{a} / \mathrm{C} 5 \mathrm{aR} 1$ axis in lung cancer dissemination and supports its potential use as a novel therapeutic target.

Keywords: complement; anaphylatoxin; aptamer; chemokine; osteoclastogensesis

(Received in original form March 31, 2017; accepted in final form January 11, 2018)

*These authors share senior authorship.

This work was supported by FIMA (Foundation for Applied Medical Research), Cancer Research Thematic Network of the Instituto de Salud Carlos III (RTICC RD12/0036/0066), CIBERONC (CB16/12/00443 and CB16/12/00350), Spanish Ministry of Economy and Competitiveness (BFU2011-22943, SAF2015-71606R,

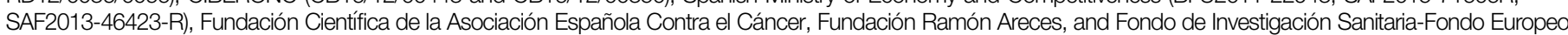

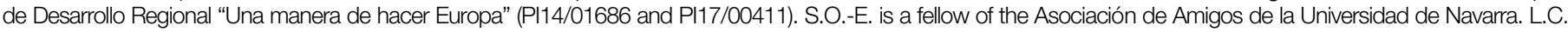

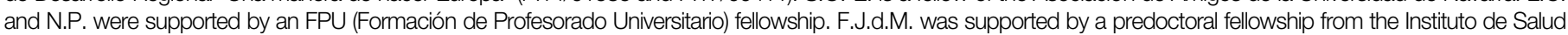

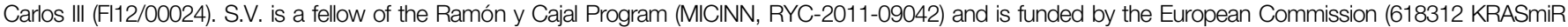
FP7-PEOPLE-2013-CIG). F.L. is funded by "la Caixa" Foundation and Caja Navarra Foundation. This work was also funded by the Foundation AECC.

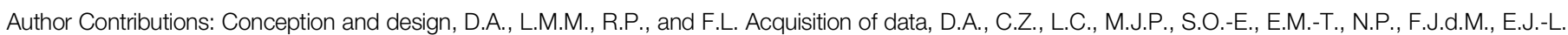

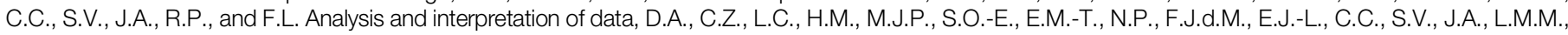

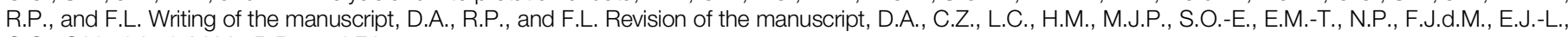
C.C., S.V., J.A., L.M.M., R.P., and F.L.

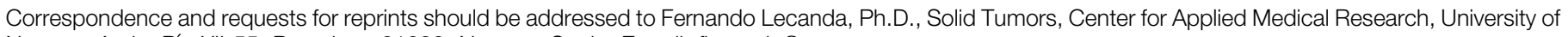
Navarra, Avda. Pío XII-55, Pamplona 31080, Navarra, Spain. E-mail: flecanda@unav.es.

This article has an online supplement, which is accessible from this issue's table of contents at www.atsjournals.org.

Am J Respir Crit Care Med Vol 197, Iss 9, pp 1164-1176, May 1, 2018

Copyright @ 2018 by the American Thoracic Society

Originally Published in Press as DOI: 10.1164/rccm.201703-06600C on January 12, 2018

Internet address: www.atsjournals.org 


\section{At a Glance Commentary}

\section{Scientific Knowledge on the}

Subject: The cleaved component of the complement system $\mathrm{C} 5 \mathrm{a}$, on binding to its receptor (C5aR1), triggers several responses that contribute to cancer progression. $\mathrm{C} 5 \mathrm{aR} 1$ is expressed in a variety of immune and tumor cell types, including lung cancer cells.

\section{What This Study Adds to the}

Field: C5aR1 expression is associated with poor prognosis in non-small cell lung cancer, and its activation increases the metastatic potential of lung cancer cells. This study suggests that $\mathrm{C} 5 \mathrm{a} / \mathrm{C} 5 \mathrm{aR} 1$ blockade may represent a novel therapeutic target to control lung cancer dissemination.

The complement system represents a central component of innate immunity. Its activation converges in the proteolytic cleavage of C3 and C5 (1). Cleaved products C3b and $\mathrm{C} 5 \mathrm{~b}$ participate in opsonization and assembly of the membrane attack complex (1), whereas the engagement of the soluble bioactive anaphylatoxins $\mathrm{C} 3 \mathrm{a}$ and $\mathrm{C} 5 \mathrm{a}$ to $\mathrm{G}$ protein-coupled receptors $\mathrm{C} 3 \mathrm{aR}$ and $\mathrm{C} 5 \mathrm{aR} 1$ (CD88) promotes a variety of processes, such as chemotaxis, vasodilation, vascular permeability, and release of reactive substances (2). C5aR1 is expressed in both myeloid and nonmyeloid cell types (2). C5a also binds to C5aR2 (GPR77 or C5L2), a receptor deficient in G-protein coupling with an uncertain function (3).

Complement components have been associated with poor prognosis in patients with lung cancer and non-small cell lung cancer (NSCLC) progression (4-7). Furthermore, neoplastic transformation entails an increased ability of lung tumor cells to overcome complement-mediated attack by a variety of mechanisms, including the secretion of soluble complement regulators $(8,9)$. C5a hampers antitumor immune responses through multiple mechanisms including the recruitment of myeloidderived suppressor cells into the tumor microenvironment $(1,4,10)$, the modulation of regulatory $\mathrm{T}$ cells, and the expression of a variety of immunosuppressive cytokines, resulting in the suppression of T-cell mediated antitumor immunity $(11,12)$.
Genetic and pharmacologic inhibition of complement was found to impair endothelial cell function in models of tumor-associated angiogenesis and to ablate ovarian cancer neovascularization (13).

All these studies underscore the emerging role played by $\mathrm{C} 5 \mathrm{a} / \mathrm{C} 5 \mathrm{aR} 1$ signaling in the tumor microenvironment, but the role of C5aR1 in tumor cells is poorly understood despite the fact that its expression has been associated with poor prognosis in a variety of human cancers (7, 14-16). Some reports have indicated that $\mathrm{C} 5 \mathrm{aR} 1$ enhances motility and invasiveness but its contribution to metastatic spread remains mostly unknown (15, 17-19).

Bone is a preferred site of metastasis in lung and other solid tumors, an event associated with dismal prognosis $(20,21)$. On engraftment in the osseous site, tumor cells release cytokines and other factors that promote high osteoclastic and proteolytic activities favoring the emergence of osteolytic lesions associated with significant morbidity (22-24).

These incipient micrometastases are exacerbated by a paracrine loop established between bone matrix-derived factors stocked in the bone compartment and released after matrix degradation by osteoclasts, and the subsequent stimulation of tumor cells $(25,26)$. Bone osteolysis ultimately results in deleterious effects on skeletal integrity and poor clinical survival (27).

Osteoclasts can activate complement by cleaving $\mathrm{C} 3$ and $\mathrm{C} 5$, releasing $\mathrm{C} 3 \mathrm{a}$ and $\mathrm{C} 5 \mathrm{a}$ and inducing osteoclastogenesis and a proinflammatory response (28). Moreover, myeloid-derived suppressor cells, which have been found to be attracted by $\mathrm{C} 5 \mathrm{a}$, can also act as osteoclast progenitors, further enhancing osteolysis $(29,30)$. Based on these findings, we postulated that the complement system may be relevant in the development of skeletal metastases in NSCLC. We show that C5aR1 levels in lung tumor cells are associated with poor prognosis in NSCLC patients and that this receptor endows tumor cells with a prometastatic phenotype. Thus, our findings unveil $\mathrm{C} 5 \mathrm{aR} 1$ as a central component in skeletal metastases, and underscore its potential as a target in cancer therapy. Some of the results of these studies have been previously reported in the form of an abstract (31).

\section{Methods}

\section{Patients and Tissue Samples}

In silico analysis was performed with patients with lung adenocarcinoma and squamous cell carcinoma included in Kaplan-Meier Plotter (http://kmplot. com/analysis/), a web-based tool that uses transcriptomic data. For immunohistochemical analysis, two cohorts of primary lung tumors were included in this study. A first set of primary tumors was obtained from 75 patients with untreated NSCLC (both adenocarcinomas and squamous cell carcinomas) at the Clínica Universidad de Navarra. A second set of primary tumors was obtained from 20 patients with NSCLC at the Hospital General Universitario de Valencia. During follow-up, bone metastases were detected in nine patients, whereas the other 11 developed metastases to other nonskeletal sites. Characteristics of Clínica Universidad de Navarra and Hospital General Universitario de Valencia cohorts are specified in Tables E1 and E2 in the online supplement, respectively. Lung primary tumors were fixed in $10 \%$ buffered formalin after surgical removal and paraffinembedded using standard procedures. Tumors were classified according to the World Health Organization 2004 classification and the International System for Staging Lung Cancer (32). For survival analysis, recurrence-free survival (RFS) and overall survival (OS) were calculated from the date of surgery to the date of recurrence or death according to the clinical information. For the evaluation, an $\mathrm{H}$-score was calculated after immunohistochemical analysis by two expert pathologists in blind records. Patients were stratified into two groups according the lower tercile of the C5aR1 $\mathrm{H}$-score. The protocol was approved by the Research Ethics Committee of the University of Navarra (Reference 108-2013) and all patients gave written informed consent.

\section{Cell Lines and Reagents}

Lung cancer cell lines were obtained from the American Type Culture Collection and were grown in RPMI 1640 with GlutaMAX (Invitrogen) supplemented with 10\% FetalClone (Thermo), penicillin, and streptomycin (Invitrogen). A549M1 and H460M5 subpopulations with propensity to form skeletal metastases were obtained 
A
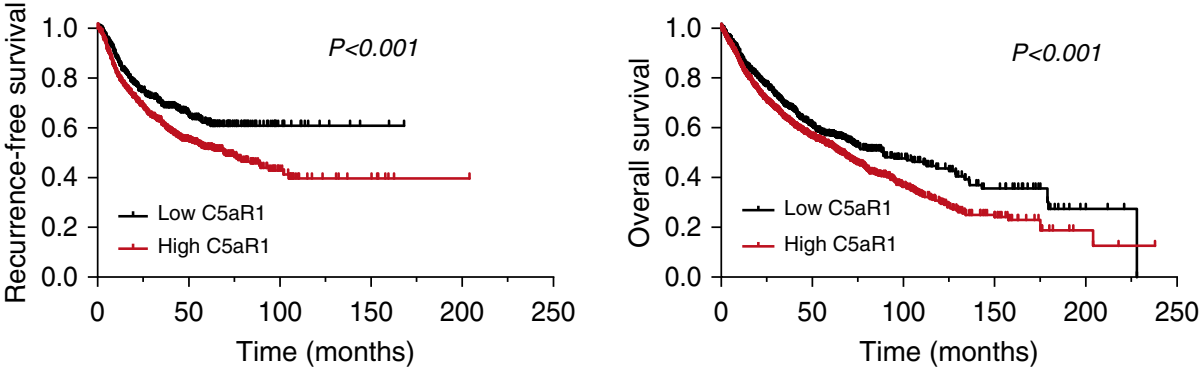

$\begin{array}{rrrrrr}\text { No. at risk } & & & & & \\ \text { Low } & 326 & 146 & 14 & 2 & 0 \\ \text { High } & 656 & 224 & 30 & 7 & 1\end{array}$

$\begin{array}{rccccc}\text { No. at risk } & & & & & \\ \text { Low } & 636 & 287 & 73 & 26 & 4 \\ \text { High } & 1,290 & 541 & 130 & 31 & 3\end{array}$

B

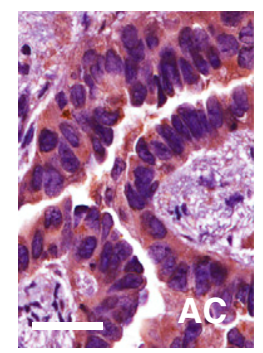

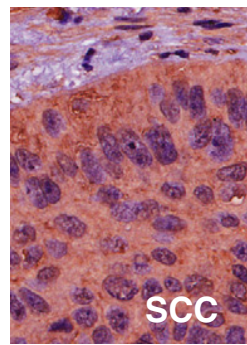

C

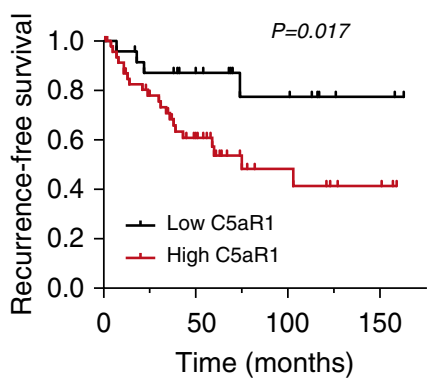

No. at risk

$\begin{array}{lllll}\text { Low } & 25 & 16 & 9 & 3 \\ \text { High } & 50 & 22 & 8 & 4\end{array}$

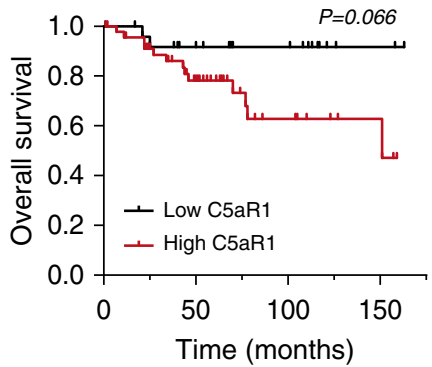

No. at risk

$\begin{array}{lllll}\text { Low } & 25 & 18 & 14 & 3\end{array}$

$\begin{array}{llll}\text { High } \quad 50 \quad 28 & 10 & 5\end{array}$

D

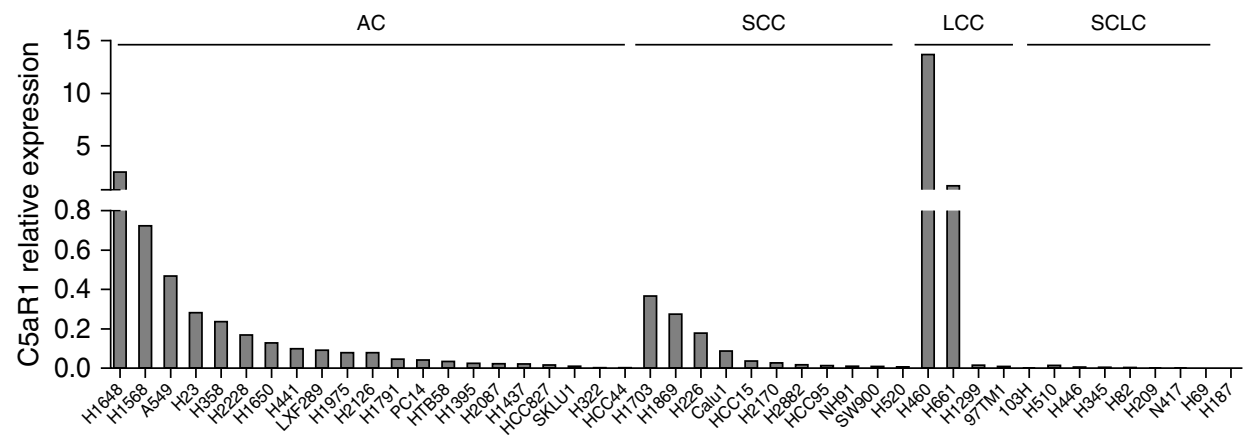

E

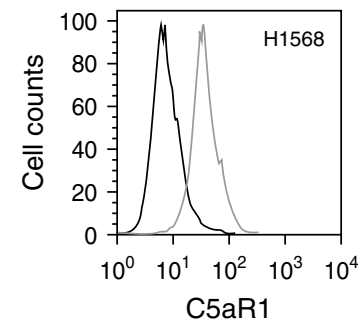

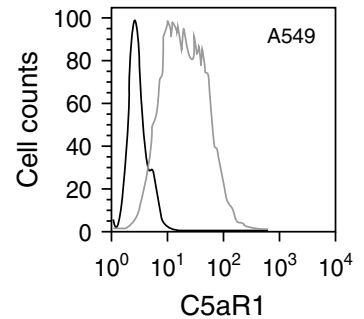

C5aR1

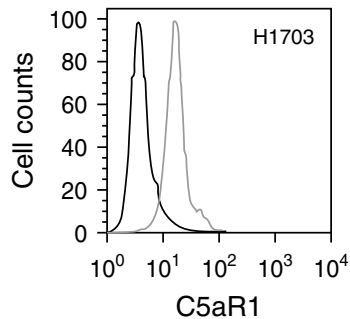

F

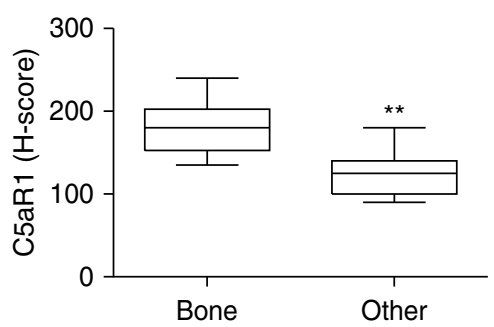

Figure 1. Expression of C5aR1 in lung cancer cells. (A) In silico analysis of the prognostic value of C5aR1 mRNA expression using Kaplan-Meier Plotter (www.kmplot.com). Patients were stratified into two groups according to the first tercile (Affy ID: 220088_at). Differences between groups were evaluated using the log-rank test. $(B)$ Representative images of C5aR1 immunostaining in human lung adenocarcinoma and squamous cell carcinoma. Scale bar = $30 \mu \mathrm{m} .(C)$ Kaplan-Meier survival curves for high and low tumor C5aR1 levels assessed by immunohistochemistry in 75 patients with non-small cell lung 
as described previously $(23,27)$. ST-2 murine stromal cells (kind gift from Dr. Civitelli, Washington University) were grown using Dulbecco's modified Eagle medium (Invitrogen) supplemented with $10 \%$ FetalClone. Human C5a was purchased from Calbiochem. Human CXCL16 was purchased from PeproTech. Both the cyclic hexapeptide $\operatorname{AcF}(\mathrm{OP}(\mathrm{D})$ ChaWR), a C5aR1 antagonist, and the corresponding control peptide were a gift from Dr. J. D. Lambris (University of Pennsylvania). AON-D21 is a PEGmodified L-aptamer (Spiegelmer), which binds to mouse/human C5 and C5a and specifically inhibits the interaction of C5a with its receptors without affecting C5 cleavage $(33,34)$. RevAON-D21 is a Spiegelmer of the reverse AON-D21 sequence that does not bind C5 or C5a. AON-D21 and revAON-D21 were provided by Aptarion biotech (Berlin, Germany). Lentiviral transductions of shRNAs against C5aR1 (TRCN0000356727 and TRCN0000008157) and a scrambled shRNA (control) from Sigma were performed as described (27).

\section{In Vivo Assays}

All animal experiments were performed according to the approved protocols of the local Animal Committee. Intracardiac (i.c.) inoculation and intratibial injection procedures, together with radiographic microcomputed tomographic analysis and bioluminescence imaging (BLI), have been previously described (35). Subcutaneous inoculation $\left(10^{6}\right.$ cells $/ 100 \mu l$ phosphate-buffered saline) was performed into the back of 8-week-old Rag2 $2^{-1-} \gamma \mathrm{c}^{-/-}$mice. AON-D21 and revAON-D21 were administered intraperitoneally $(10 \mathrm{mg} / \mathrm{kg})$ in saline every other day starting 1 day before intracardiac injection of A549M1 cells. Tumor volume was measured with a digital caliper and calculated as previously described (25).

\section{Statistical Analysis}

Statistical analysis was performed using SPSS 15.0 (IBM). To study differences between experimental groups, data were analyzed using the Mann-Whitney $U$ test or Kruskal-Wallis test. In silico analysis was performed with both lung adenocarcinoma and squamous cell carcinoma patients using Kaplan-Meier Plotter (www.kmplot. com), with the probeset Affy ID: 220088_at. Kaplan-Meier survival curves and the logrank test were used to analyze differences in survival time. Patients were stratified into two groups according to the first $\mathrm{C} 5 \mathrm{aR} 1$ expression tercile. The univariate and multivariate Cox proportional hazards modeling was used to determine the effects of variables on RFS and OS. Only those variables with $P$ less than 0.1 in the univariate analysis were included in the multivariable analysis. The proportional hazards assumption was examined by testing interactions between the covariables of the final model and time. Statistical significance was defined as two-tailed $P$ less than $0.05, P$ less than 0.01 , or $P$ less than 0.001 . All the rest of methods including in vitro assays appear in the online supplement.

\section{Results}

\section{C5aR1 Is Expressed in Lung Cancer Cells}

First, we explored the prognostic value of C5aR1 expression in NSCLC using the in silico tool Kaplan-Meier Plotter. Patients were classified into two groups according to their C5aR1 mRNA levels in primary tumors. Patients with high levels of C5aR1 had significantly shorter RFS and OS $(P<$ 0.001 ) (Figure 1A). Immunohistochemical analysis of patients with NSCLC $(n=75)$ from the Clínica Universidad de Navarra showed positive staining in stromal and epithelial cells in the alveolar parenchyma, bronchi, and bronchioles, and in neutrophils and macrophages (see Figure E1). C5aR1 expression was also found in lung tumor cells (Figure 1B). Interestingly, RFS was significantly decreased in patients with high $\mathrm{C} 5 \mathrm{aR} 1$ expression in the tumor cells $(P=0.017)$ (Figure 1C). A similar trend was observed for OS, although without reaching statistical significance $(P=0.066)$. A multivariable analysis, adjusted for sex, age, smoking status, and stage, showed that high C5aR1 expression was an independent prognostic factor for RFS in patients with NSCLC (hazard ratio, 3.187; 95\% confidence interval, 1.079-9.411; $P=0.036$ ) (Table 1). A trend toward significance was observed for OS (hazard ratio, 4.032; 95\% confidence interval, 0.887-18.325; $P=0.071$ ).

Next, we evaluated the expression levels of C5aR1 in a panel of 45 cell lines representing the main lung cancer subtypes. Many of them expressed C5aR1 mRNA, although the amount of mRNA was variable between cell lines (Figure 1D). C5aR1 was also detected by flow cytometry on the cell surface of representative lung cancer cell lines (Figure 1E). Interestingly, on a preliminary observation, we found significantly higher C5aR1 levels in primary NSCLC tumors from patients who developed bone metastases during disease progression as compared with tumors from patients who developed metastases to other nonskeletal sites (Figure 1F).

Moreover, addition of $\mathrm{C} 5 \mathrm{a}$ to cultured cells led to phosphorylation of p42/44 MAPK, and translocation of nuclear factor $-\kappa \mathrm{B}$ to the nucleus (see Figures E2A and $\mathrm{E} 2 \mathrm{~B}$ ). We next evaluated the expression of a panel of cytokines and growth factors (Luminex technology), which included known targets of nuclear factor- $\mathrm{kB}$, such as IL-8 (36), in the supernatant of A549 cells incubated with C5a. An increase in the levels of IL-8, monocyte chemotactic protein 1, and vascular endothelial growth factor was observed, whereas no changes were found in the levels of epidermal growth factor (see Figure E2C). These results suggest that lung cancer cells express a functional C5aR1 that may contribute to NSCLC progression.

Figure 1. (Continued). cancer. Patients were stratified into two groups according to the first tercile, and differences between groups were evaluated using the log-rank test. (D) C5aR1 mRNA expression in a panel of 45 lung cancer cell lines determined by real-time PCR (IPO 8 was used as the reference gene). (E) C5aR1 cell membrane expression assessed by flow cytometry in $\mathrm{H} 1568$, A549, and $\mathrm{H} 1703$ lung cancer cell lines. Incubation of cells without the primary antibody was used as negative control (dark line). ( $F$ ) Immunohistochemical analysis of C5aR1 in primary tumors from nine patients with resectable non-small cell lung cancer who developed (at relapse) bone metastases (Bone) and 11 patients with extraskeletal metastases (Other). Data are presented as minimum to maximum box-and-whisker plots. ${ }^{\star \star} P<0.01 . \mathrm{AC}=$ adenocarcinoma; C5aR1 = receptor 1 for cleaved complement component of $\mathrm{C} 5$; $\mathrm{LCC}=$ large cell carcinoma; $\mathrm{SCC}=$ squamous cell carcinoma; $\mathrm{SCLC}=$ small cell lung cancer. 
Table 1. Univariate and Multivariate Cox Regression Analyses of C5aR1 Protein Expression for RFS and OS in Patients with NSCLC

\begin{tabular}{|c|c|c|c|c|c|c|c|c|c|}
\hline \multirow[b]{2}{*}{ C5aR1 } & \multirow[b]{2}{*}{$n$} & \multicolumn{4}{|c|}{ RFS } & \multicolumn{4}{|c|}{ os } \\
\hline & & $\begin{array}{l}\text { Crude HR } \\
(95 \% \mathrm{Cl})\end{array}$ & $P$ Value & $\begin{array}{c}\text { Adjusted HR } \\
(95 \% \mathrm{Cl})^{*}\end{array}$ & $\begin{array}{l}\text { Adjusted } \\
P \text { Value }\end{array}$ & $\begin{array}{l}\text { Crude HR } \\
\text { (95\% Cl) }\end{array}$ & $P$ Value & $\begin{array}{l}\text { Adjusted HR } \\
(95 \% \mathrm{Cl})^{*}\end{array}$ & $\begin{array}{l}\text { Adjusted } \\
P \text { Value }\end{array}$ \\
\hline Low & 25 & & & & & & & & \\
\hline High & 50 & $\begin{array}{c}3.542 \\
(1.209-10.380)\end{array}$ & 0.021 & $\begin{array}{c}3.187 \\
(1.079-9.411)\end{array}$ & 0.036 & $\begin{array}{c}3.665 \\
(0.826-16.262)\end{array}$ & 0.088 & $\begin{array}{c}4.032 \\
(0.887-18.325)\end{array}$ & 0.071 \\
\hline
\end{tabular}

Definition of abbreviations: C5aR1 = receptor 1 for cleaved complement component of $\mathrm{C} 5 ; \mathrm{Cl}=$ confidence interval; $\mathrm{HR}=$ hazard ratio; $\mathrm{NSCLC}=$ non-small cell lung cancer; OS = overall survival; RFS = recurrence-free survival

${ }^{*}$ Adjusted by sex, stage, smoking status, and age.

\section{The C5a/C5aR1 Axis Has a Role in Lung Cancer Cell Migration and Invasion}

We evaluated the in vitro effects mediated by $\mathrm{C} 5 \mathrm{aR} 1$ activation on lung cancer cell growth. Incubation of A549 cells with recombinant $\mathrm{C} 5 \mathrm{a}$ did not affect in vitro proliferation or cell cycle distribution (see Figures E3A and E3B). However, in the scratch wound healing assay, recombinant C5a significantly increased the motility of A549 cells in a dose-dependent manner (Figure 2A). C5a also increased Matrigel invasiveness of A549 cells (Figure 2B). Similar results were obtained with the human lung large cell carcinoma cell line H460M5 (see Figure E4).

Because invasiveness has been linked to proteolytic matrix degradation, we evaluated whether C5aR1 activation could modify extracellular matrix metalloproteinase (MMP) activity. Conditioned culture media from C5aactivated A549 cells showed a significant increase in metalloproteolytic activity as evaluated by cleavage of a fluorogenic peptide (Figure 2C). MMP activity was inhibited by the global MMP inhibitor GM6001 (data not shown). Moreover, A549 adhesion to collagen I and IV was significantly reduced when cells were treated with C5a. This effect was not observed using fibronectin and vitronectin matrices (see Figure E5). Taken together, these findings indicate that $\mathrm{C} 5 \mathrm{a}$ induces lung cancer cell motility, invasiveness, and MMP activity, and reduces cell-matrix adhesion, without affecting cell growth kinetics in vitro.

\section{C5aR1 Knockdown in Lung Cancer Cells Prevents Bone Metastasis}

Taking into account that migration and invasion are critical events for metastatic progression, we sought to investigate the contribution of the C5a/C5aR1 axis to the prometastatic activity of lung cancer cells. For that purpose, we used a human lung adenocarcinoma subpopulation (A549M1) derived from parental A549 cells and previously described for its proclivity to selectively form osseous metastases (23). In these cells, we stably silenced C5aR1 expression using lentiviral transduction of two different shRNAs, shC5aR1-1 and shC5aR1-2 (Figure 2D). As expected, there were no significant differences in in vitro proliferation and apoptosis between cells transduced with C5aR1-specific shRNAs and a scrambled shRNA used as control (see Figures E3C and E3D). However, the capacity of $\mathrm{C} 5 \mathrm{a}$ to induce the motility or invasiveness of A549M1 cells was impaired by knocking down $\mathrm{C} 5 \mathrm{aR} 1$ expression (Figures 2E and 2F), and shC5aR1-silenced cells showed lower metalloproteolytic activity than control cells (Figure 2G).

We next tested whether C5aR1silenced cells displayed an impaired metastatic activity. We used two different in vivo models based on tail vein injection (lung metastasis model) or left ventricle inoculation (bone metastasis model) of A549M1 cells. Although downregulation of C5aR1 did not affect in vivo growth of primary subcutaneous tumors from shC5aR1-transduced A549M1 cells (see Figure E3E), mice intravenously injected with A549M1 shC5aR1 cells showed a decrease in lung metastatic burden as compared with those injected with parental cells (see Figure E6). The intracardiac inoculation of these cells, as outlined in Figure 3A, resulted in even more drastic effects. C5aR1-silencing led to a delayed appearance of signs of morbidity, including tumor-induced cachexia and reduced mobility, as compared with the administration of control cells (Figure 3B). Consistently, BLI showed a significantly decreased tumor burden in mice inoculated with shC5aR1-transduced cells (Figures 3C and $3 \mathrm{~F}$ ). A decrease in bone metastatic area was observed by X-ray imaging (Figures 3D and $3 \mathrm{G}$, top row). Microcomputed tomography scans and histologic analyses revealed a similar decrease in tumor burden (Figures $3 \mathrm{E}$ and 3G, middle and bottom rows). No differences in the number of apoptotic cells were detected by cleaved caspase- 3 immunostaining in metastatic tumors (data not shown). However, the percentage of proliferating $\mathrm{Ki}^{+} 7^{+}$cells observed in bone metastases was significantly lower in mice inoculated with shC5aR1-silenced cells (Figure 3H).

Silenced C5aR1 expression levels were maintained along the whole experimental period as evaluated by immunohistochemistry (Figure 3I). We extended these results to H460M5 cells, which showed high C5aR1 expression levels and generate highly aggressive osteolytic lesions with high bone tropism (23). The intracardiac inoculation of shC5aR1transduced H460M5 cells resulted also in a significant decrease of tumor burden as compared with control cells (see Figure E7). Taken together, these data indicate that C5aR1 signaling promotes the metastasis of lung cancer cells.

\section{C5aR1 Expression on Lung Cancer Cells Promotes Osseous Colonization}

To assess the contribution of C5aR1 to cell growth and colonization within the osseous compartment, control and shC5aR1-

silenced A549M1 cells were directly injected into the tibiae (Figure 4A). Twenty days after injection, BLI showed an overt decrease in tibiae tumor burden in mice inoculated with shC5aR1-kockdown cells as compared with control cell-injected mice (Figure 4B). Accordingly, X-ray radiography, microcomputed tomography 
A

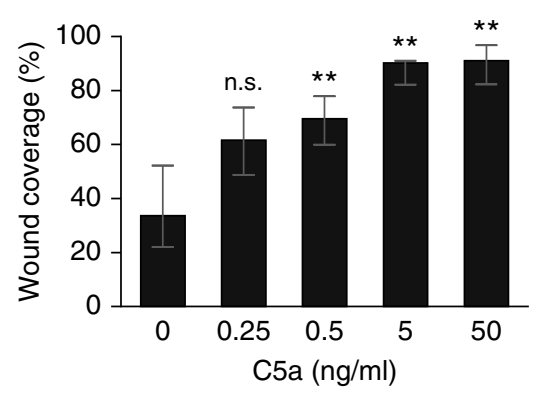

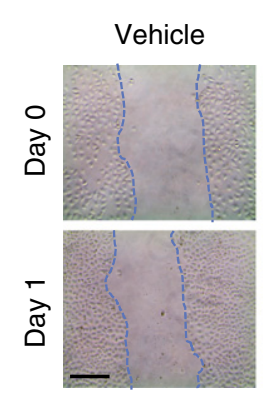

D
B

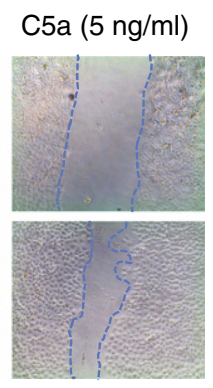

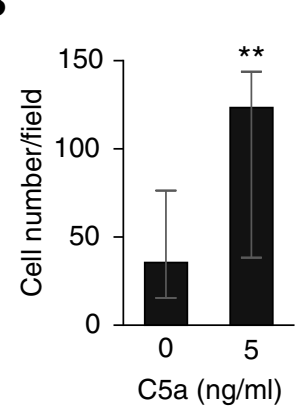

Vehicle

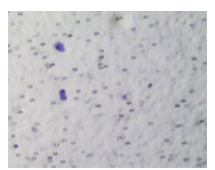

$\mathrm{C} 5 \mathrm{a}(5 \mathrm{ng} / \mathrm{ml})$

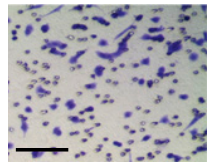

E

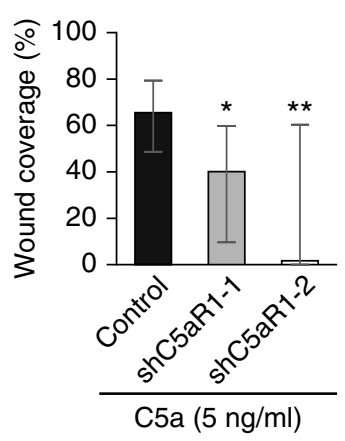

C

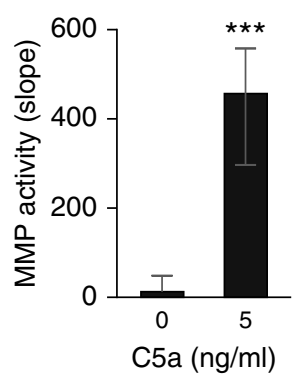

E

G

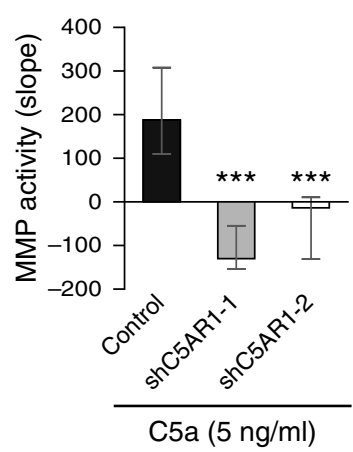

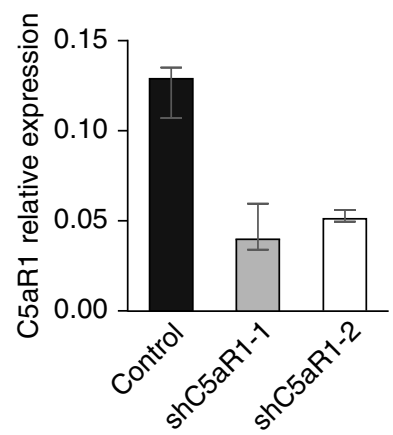
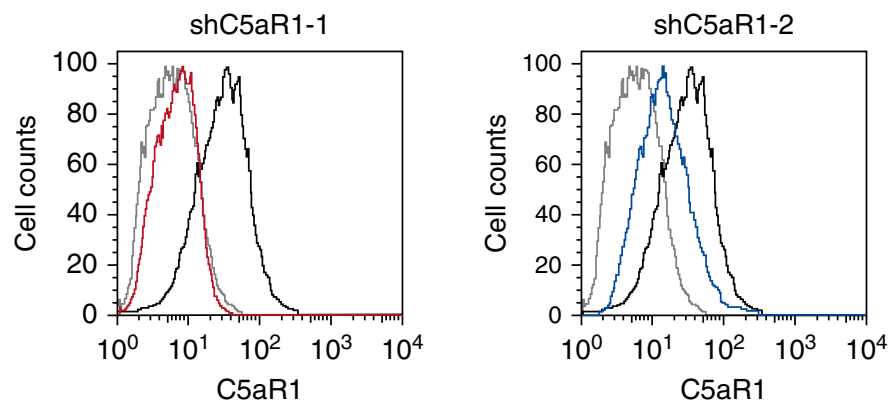

F

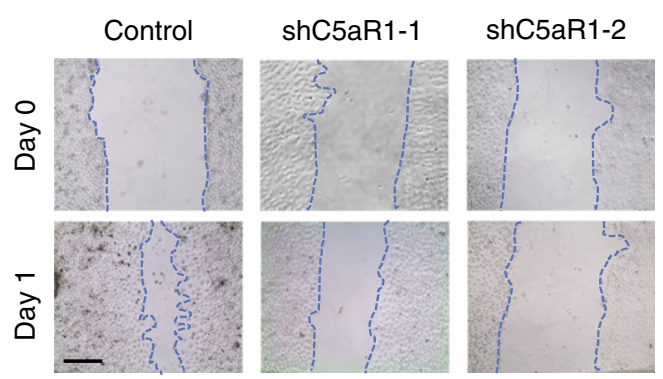

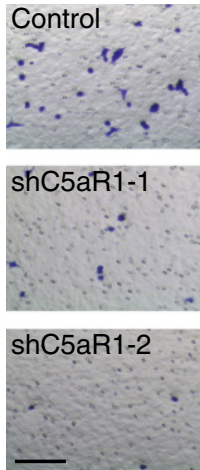

Figure 2. Role of $\mathrm{C} 5 \mathrm{a} / \mathrm{C} 5 \mathrm{RR} 1$ in migration, invasion, and tumor-associated matrix metalloproteinase (MMP) activity. (A) Scratch wound healing assay after incubation of A549 cells with recombinant C5a. The percentage of the area covered by migrated cells (wound coverage) was calculated. (B) Invasion assay of A549 cells after treatment with recombinant C5a. Cells were counted in four independent fields of the bottom side of the Boyden chamber after crystal violet staining. (C) MMP activity in the serum-free conditioned medium of A549 cells after treatment with recombinant C5a. MMP activity was assessed by digestion of a fluorogenic substrate. (D) Left: Gene expression levels of C5aR1 were silenced in A549M1 lung cancer cells using two lentiviral transduced shRNAs targeting two different sequences (shC5aR1-1 and shC5aR1-2). Scrambled shRNA-transduced cells were used as control. IPO8 was used as the reference gene. Right: Flow 
scans (Figures 4C and 4E, top and middle rows), and image analysis of histologic sections of hindlimbs (Figures 4D and 4E, bottom row) showed a decreased tumor area in shC5aR1-injected mice as compared with control animals.

To test whether differences in tumor burden were caused by change in cell growth kinetics within the osseous microenvironment, immunohistochemical staining of Ki67 and cleaved caspase- 3 were performed. No differences in the number of apoptotic cells were observed (data not shown), but a lower percentage of $\mathrm{Ki}^{+}{ }^{+}$ cells was detected in shC5aR1-silenced tumors (Figure 4F).

Tumor-induced osteoclastogenesis is a major mechanism of bone degradation in osseous metastases (20). Thus, we studied whether the reduction of osteolytic lesions in our in vivo model was associated with an impaired osteoclastogenic activity. Histochemical distribution of tartrateresistant acid phosphatase (TRAP) staining was used to identify osteoclasts. Analysis of tibiae harboring shC5aR1-knockdown cells revealed a dramatic decrease of $\mathrm{TRAP}^{+}$multinucleated cells at the tumorbone interphase (Figure 4G).

\section{Pharmacologic Inhibition of C5a Decreases Bone Metastases}

To obtain pharmacologic evidence of the role played by $\mathrm{C} 5 \mathrm{a} / \mathrm{C} 5 \mathrm{aR} 1$ in metastasis and to address the potential therapeutic utility of targeting this signaling pathway, we used a plasma stable anti-C5a L-aptamer, AON-D21, in the in vivo bone metastasis model of A549M1 cells, following the schedule outlined in Figure 5A. A non-C5abinding L-oligonucleotide of the same length but the reverse nucleotide sequence, revAON-D21, was used as negative control. Twenty-six days after intracardiac inoculation of metastatic A549M1 cells, BLI, X-ray imaging, histologic analysis, and microcomputed tomography scan analysis demonstrated a marked decrease of tumor burden in hindlimbs of AON-D21treated mice as compared with revAON-
D21-treated mice (Figures 5B-5F). This finding emphasizes the relevance of C5a/C5aR1 signaling as an effective pharmacologic target to suppress bone metastasis of lung cancer cells.

Taken together, these data suggest that C5a through C5aR1 on lung cancer cells contributes to tumor expansion and osseous colonization by inducing osteolysis, an event highly dependent on the hosting milieu.

\section{Silencing C5aR1 or Intracellular C5 Levels Decreases CXCL16 Expression}

We further studied the mechanism by which C5aR1 knockdown regulates osseous colonization. Considering that chemokines play a prominent role in the metastatic spread of several human malignancies including lung cancer (37), we focused our attention in CXCL16, a chemokine that induces A549 migration and invasion (38), supports lung cancer metastatic process by increasing metalloproteinase activity (39), and is highly expressed in specimens of bone metastasis $(40,41)$. By real-time PCR analysis, we found that CXCL16 is downregulated in A549M1 (Figure 6A, left panel) and H460M5 (see Figure E6E) shC5aR1-silenced cells. Consistently, the protein levels of CXCL16 secreted by A549M1 C5aR1-silenced cells were diminished in comparison with control cells (Figure 6A, right panel). However, exogenous addition of $\mathrm{C} 5 \mathrm{a}$ did not modify the levels of CXCL16 in A549M1 cells (data not shown).

Intracellular conversion of $\mathrm{C} 5$ to $\mathrm{C} 5 \mathrm{a}$, and $\mathrm{C} 5 \mathrm{aR} 1$ activation, has been described in some cell types (42). Therefore, we explored whether intracellular signaling was responsible for the modulation of CXCL16 in lung cancer cells. Interestingly, silencing of endogenous C5 levels by transduction of two independent specific siRNAs (Figure 6B, left panel) led to a significant decrease in CXCL16 expression levels (Figure 6B, right panel), suggesting that intracellular $\mathrm{C} 5$, likely by its activation to C5a, also signals through C5aR1.
Interestingly, extracellular addition of $\mathrm{C} 5$ did not rescue the levels of CXCL16 in these cells (data not shown). These findings suggest that extracellular and intracellular signaling are functional in $\mathrm{C} 5 \mathrm{aR} 1-$ expressing lung tumor cells.

\section{Effect of C5aR1 on Osteoclastogenesis In Vitro}

To further study the mechanisms of tumor-driven C5aR1-dependent osteolysis, we explored whether C5aR1 signaling would result in the secretion of soluble factors that drive osteoclast differentiation. For this purpose, we performed an in vitro osteoclastogenesis assay in which macrophage colony-stimulating factor and RANKL, together with the conditioned medium of A549M1 cells cocultured with the bone stromal cell line ST-2, were used to differentiate murine bone marrow mononuclear precursors into mature osteoclasts. For this experiment, we relied on the endogenous production of $\mathrm{C} 5 \mathrm{a}$ by the A549M1 cells (4), avoiding the addition of exogenous C5a to minimize its direct positive effect on osteoclastogenesis (28). Incubation of osteoclast precursor cells with the conditioned medium derived from shC5aR1-silenced cells led to a decrease in the area of multinucleated $\mathrm{TRAP}^{+}$osteoclasts, as compared with the incubation with conditioned medium from control cells (Figure 6C).

To establish the specific role of CXCL16 levels in osteoclastogenesis, beyond other proosteoclastogenic cytokines released by C5a (see Figure E2C), we incubated murine bone marrow mononuclear cells with macrophage colony-stimulating factor and RANKL, together with increasing concentrations of CXCL16. Interestingly, CXCL16 enhanced the area of multinucleated TRAP $^{+}$cells in a dosedependent manner (Figure 6D). More importantly, the addition of CXCL16 to the conditioned medium from shC5aR1silenced A549M1 cells rescued its ability to generate osteoclasts (Figure 6E). These findings indicate that $\mathrm{C} 5 \mathrm{aR} 1-\mathrm{knockdown}$

Figure 2. (Continued). cytometry analysis of the membrane expression of C5aR1 in shC5aR1-1 (red line), shC5aR1-2 (blue line), and scrambled shRNA (black line)-transduced cells. Incubation without primary antibody was used as negative control (gray line). (E) Scratch wound healing assay of C5aR1-silenced cells (shC5aR1-1 and shC5aR1-2) and control cells after treatment with recombinant C5a. (F) Invasion assay of C5aR1-silenced and control cells after treatment with recombinant C5a. (G) MMP activity in the serum-free conditioned medium of C5aR1-silenced and control cells after treatment with recombinant C5a. Data in this figure are presented as medians and interquartile ranges. Results in $A-C$ and $E-G$ correspond to three independent experiments. Differences between treatments were analyzed with the Mann-Whitney $U$ test. Scales bars in representative images, $100 \mu \mathrm{m}$; applies to all. ${ }^{\star} P<0.05 ;{ }^{* \star} P<0.01 ;{ }^{* \star \star} P<0.001$. C5a $=$ cleaved complement component of C5; C5aR1 = receptor 1 for C5a; n.S. = non-significant; shC5aR1-1 = short hairpin-mediated silencing of C5aR1 gene expression, using shRNA-1; shC5aR1-2 = short hairpin-mediated silencing of C5aR1 gene expression, using shRNA-2. 
A

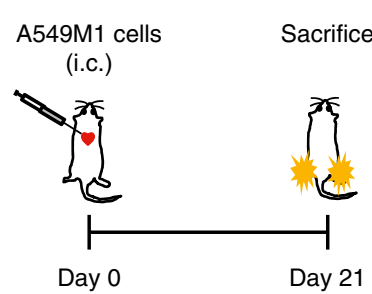

$\mathbf{F}$

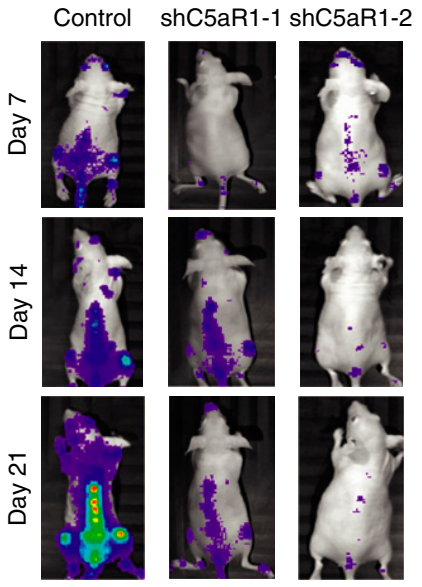

$X 10^{6}$, counts $100 \begin{array}{lllllllll}90 & 80 & 70 & 60 & 50 & 40 & 30 & 20 & 10\end{array}$
B

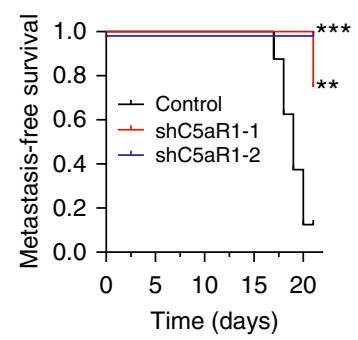

G

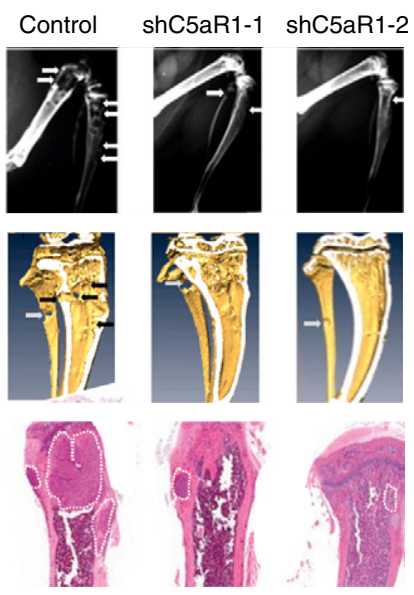

C

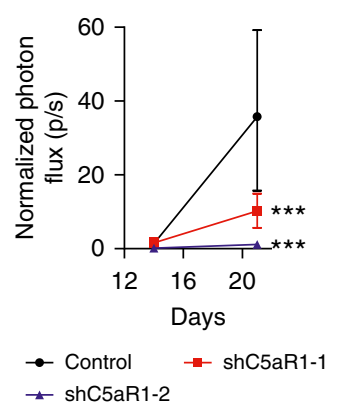

D

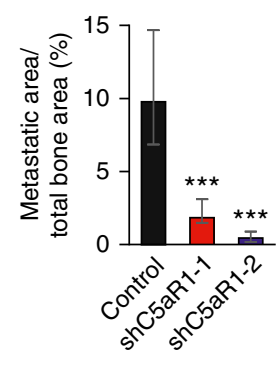

E

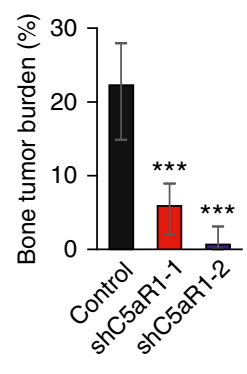

H

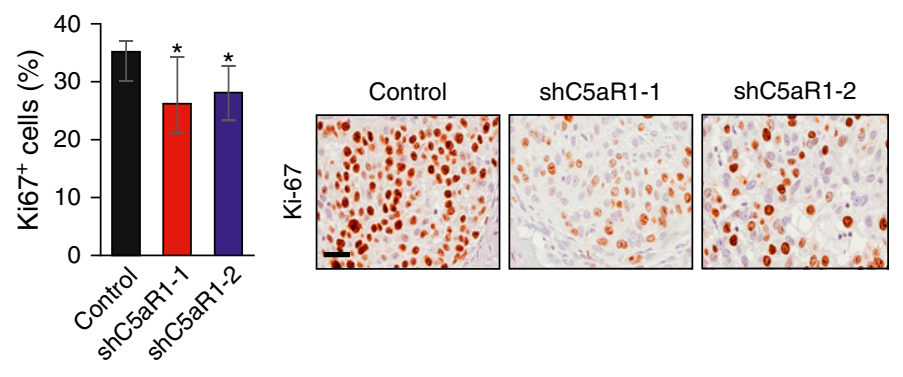

I

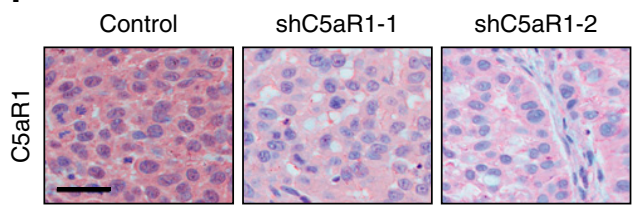

Color bar

Figure 3. Effects of C5aR1 knockdown in A549M1 bone metastasis in vivo. (A) Schematic outline of the experiment. (B) Kaplan-Meier curves of metastasis-free survival after intracardiac inoculation of control, shC5aR1-1 or shC5aR1-2 A549M1 cells (eight mice per group). Signs of morbidity (cachexia or reduced mobility) were used as the endpoint for metastasis-free survival. Differences between groups were evaluated using the log-rank test. (C) Bioluminescence imaging quantification in hindlimbs. (D) $X$-ray image analysis of metastatic area in hindlimbs on Day 21. (E) Hindlimbs tumor burden assessment by hematoxylin and eosin staining in histologic sections (Day 21). ( $F$ ) Representative images of bioluminescence imaging. (G) Representative X-ray images (top row), microcomputed tomography scans (middle row), and hematoxylin and eosin-stained histologic sections (bottom row) (Day 21). (H) Left: quantitative analysis of the percentage of Ki-67 $7^{+}$tumor cells. Right: representative Ki-67 immunostaining in metastatic lesions from C5aR1-silenced and control hindlimbs. () Representative human C5aR1 immunostaining in metastatic lesions from C5aR1-silenced and control hindlimbs. Scale bar = $30 \mu \mathrm{m}$; applies to all images. Data are presented as medians and interquartile ranges. Differences among experimental groups were analyzed using the Mann-Whitney U test. ${ }^{*} P<0.05$; ${ }^{\star *} P<0.01$; ${ }^{\star \star} P<0.001$. C5aR1 = receptor 1 for cleaved complement component of C5; i.C. = intracardiac inoculation; shC5aR1-1 = short hairpin-mediated silencing of C5aR1 gene expression, using shRNA-1; shC5aR1-2 = short hairpin-mediated silencing of C5aR1 gene expression, using shRNA-2.

cells release lower levels of CXCL16, leading to a diminished osteoclastogenic potential as compared with control cells. Thus, signaling via C5aR1 in lung cancer cells regulates CXCL16 levels, which contributes to tumor-induced osteoclastogenic activity.

\section{Discussion}

In the present study, we show that the anaphylotoxin $\mathrm{C} 5 \mathrm{a}$, through its receptor C5aR1 expressed in lung tumor cells, has a relevant role in lung cancer promotion.
Specifically, the activation of C5aR1 induces the production of osteoclastogenic factors and markedly promotes lung cancer bone metastasis. Consequently, genetic and pharmacologic abrogation of the C5a/ C5aR1 signaling results in a dramatic reduction of tumor-induced osteolysis and bone metastasis leading to increased metastasis-free survival. Our study unveils a crucial role of C5aR1 as a bona fide target to impair bone metastasis and osseous colonization in NSCLC (Figure 6F).

The prometastatic phenotype conferred by C5a binding to C5aR1 was related to an increase in tumor cell invasion, migration, MMP activity, and osteolysis. Our results suggest that the effects on osteolysis were mediated by osteoclastogenic cytokines, such as IL-8 and monocyte chemotactic protein 1 . The angiogenic factor vascular endothelial growth factor would also contribute to skeletal colonization. Moreover, we have found that CXCL16 acts concomitantly with these factors. CXCL16 signals through CXCR6, a receptor that is present in lung tumor cells (39) and is associated with inflammatory states (43). It has 
A

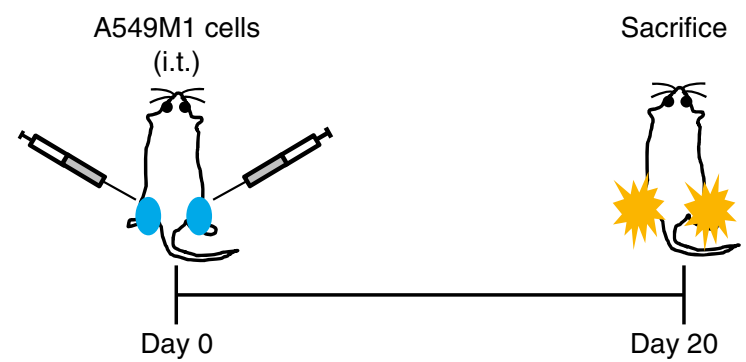

E

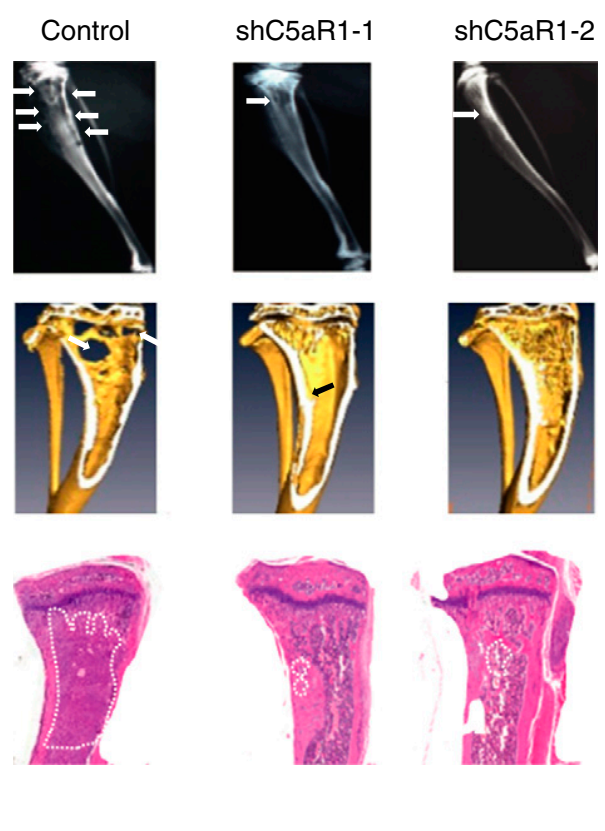

$\mathbf{F}$

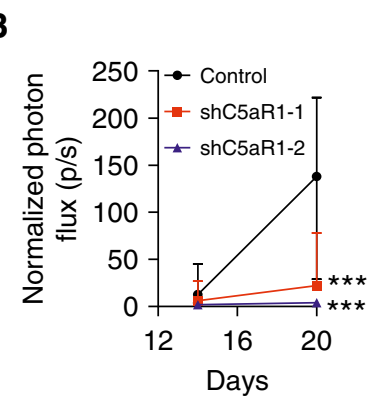

B

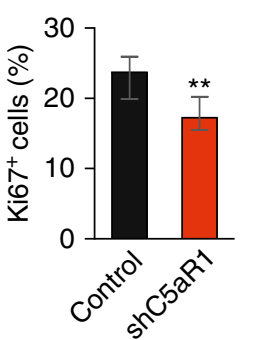

C

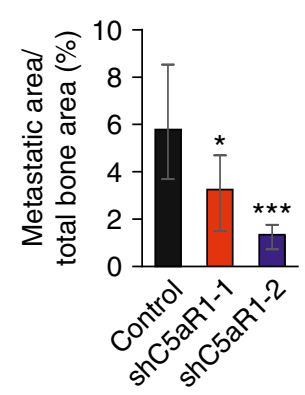

D

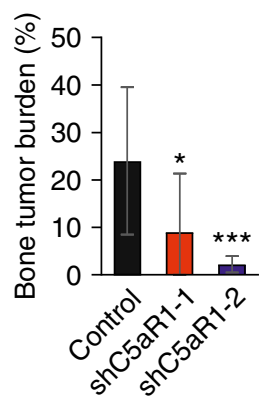

G

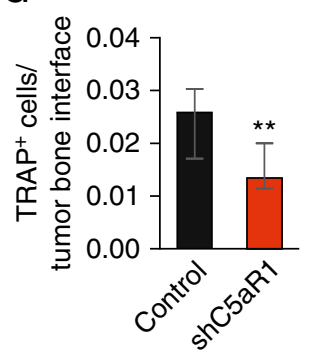

Control shC5aR1

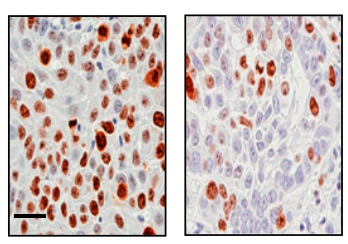

Figure 4. Abrogation of lung cancer osseous colonization by silencing C5aR1 levels. (A) Experimental regimen of bone colonization assay after tumor cell injection into the tibiae. (B) Quantification of bioluminescence imaging in control and shC5aR1-transduced cells (five to eight mice per group). (C) Assessment of tumor burden in hindlimbs of animals inoculated with shC5aR1 or control cells by X-ray image analysis at Day 20. (D) Histologic tumor burden analysis in hematoxylin and eosin-stained sections evaluated by image analysis (Day 20). (E) Representative images of X-rays (top row), microcomputed tomography (middle row), and hematoxylin and eosin staining (bottom row) (Day 20); arrows indicate osteolytic lesions. (F) Quantitative analysis and representative images of proliferation measured as the percentage of $\mathrm{Ki}-67^{+}$tumor cells per total tumor area. Scale bar $=30 \mu \mathrm{m}$; applies to all. (G) Left: Number of osteoclasts at tumor-bone interface. Right: Representative images; scale bar as in F. Arrowheads show osteoclasts. Data are presented as medians and interquartile ranges. Differences among experimental groups were analyzed using the Mann-Whitney $U$ test. ${ }^{*} P<0.05$; ${ }^{\star \star} P<0.01 ;{ }^{\star \star \star} P<0.001$. B = bone; C5aR1 = receptor 1 for cleaved complement component of C5; i.t. = intratibial; shC5aR1-1 = short hairpin-mediated silencing of C5aR1 gene expression, using shRNA-1; shC5aR1-2 = short hairpin-mediated silencing of C5aR1 gene expression, using shRNA-2; $\mathrm{T}=$ tumor; TRAP $=$ tartrate-resistant acid phosphatase .

been reported that the activation of the CXCL16/CXCR6 axis modulates tumor-induced MMP expression and increases tumor cell invasiveness and migration, favoring tumor progression (39). Moreover, CXCL16 also plays a role in skeletal metastases in prostate cancer $(40$, 41). In our model, the release of CXCL16, on activation of $\mathrm{C} 5 \mathrm{a} / \mathrm{C} 5 \mathrm{aR} 1$ signaling, would promote a proosteoclastogenic environment in bone metastasis. In a similar context, a CXCL16-induced
RANKL expression, which is characteristic for bone resorption, was also observed in synovial fibroblasts derived from patients with rheumatoid arthritis (44).

The effect of C5a/C5aR1 signaling on the intrinsic tumor cell phenotype was dependent on the host microenvironment. Thus, subcutaneous tumor growth was unaffected by C5aR1 levels, whereas the percentage of proliferating Ki67 ${ }^{+}$ cells observed in bone metastases was significantly lower after intracardiac inoculation of shC5aR1-silenced cells, and survival of these cells on direct intratibial injection was severely impaired. The expression of C5aR1 by stromal components of the metastatic milieu could also confer additional roles unexplored in our models. C5aR1 expression influences osteoclast formation by direct and indirect effects (45), and reports in syngeneic models showed that C5aR1 displays an array of immunomodulatory functions that were highly context-dependent (46). 
A
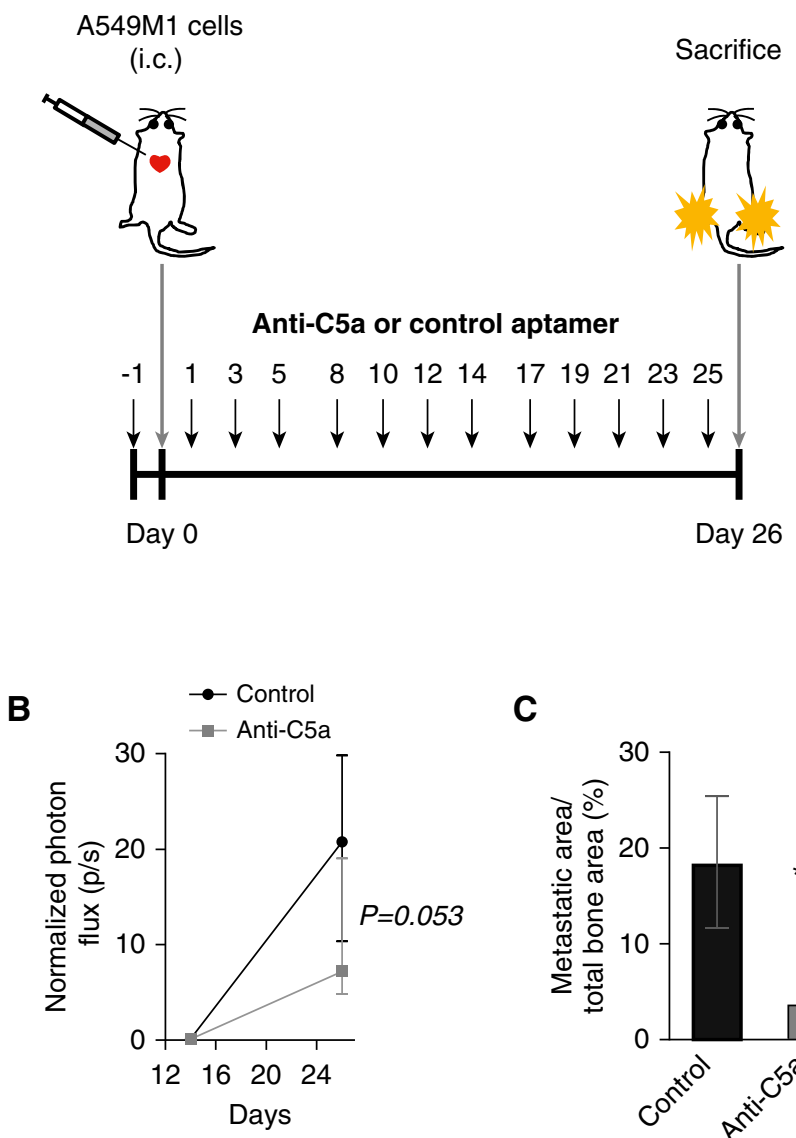

E

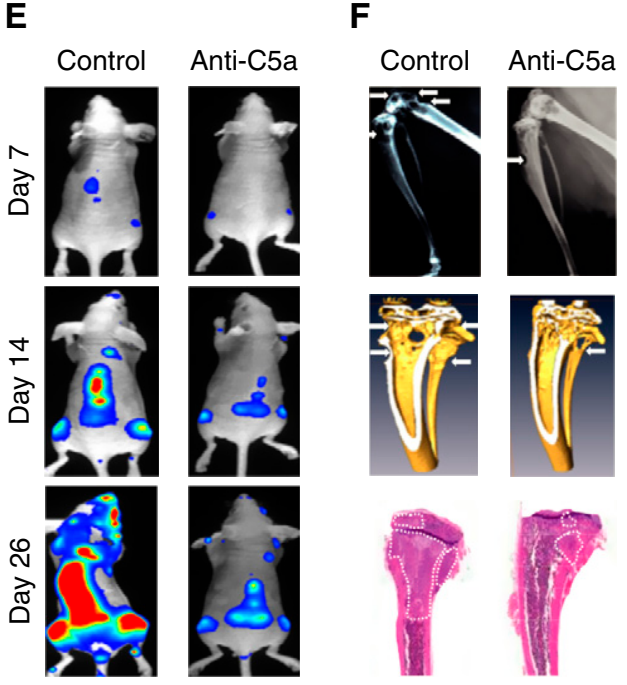

C

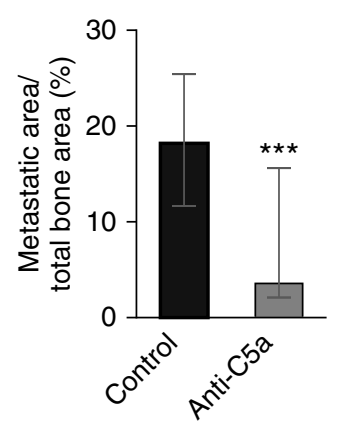

D

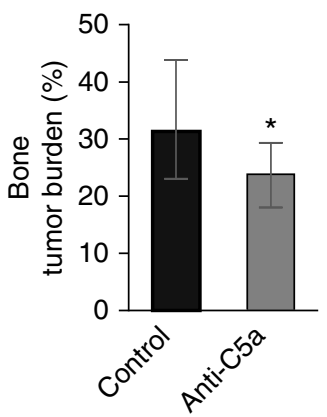

Figure 5. Effect of blocking C5a on bone metastasis formation of A549M1 cells. (A) Experimental regimen for the treatment of A549M1-injected mice with $10 \mathrm{mg} / \mathrm{kg}$ intraperitoneally of the anti-C5a L-aptamer AON-D21 $(n=7)$ or the control aptamer revAON-D21 $(n=8)$. (B) Quantification of BLI in hindlimbs 26 days after intracardiac inoculation. $(C)$ Quantification of metastatic area by X-ray imaging. (D) Tumor burden assessed in hematoxylin and eosin-stained sections (Day 26). (E) Representative images of bioluminescence imaging (Day 26). ( $F$ ) Representative X-ray images (top row), microcomputed tomography scans (middle row), and hematoxylin and eosin-stained histologic sections (bottom row) of each group (Day 26); arrows indicate osteolytic lesions. Data are presented as medians and interquartile ranges. Differences among experimental groups were analyzed using the Mann-Whitney $U$ test. ${ }^{\star} P<0.05 ;{ }^{\star \star} P<0.001$. C5a = cleaved complement component of C5; i.c. $=$ intracardiac.

Interestingly, C5a/C5aR1 signaling contributes to tumor progression and metastasis by suppressing T-cell responses and recruiting myeloid-derived suppressor cells and regulatory $\mathrm{T}$ cells, which regulate the secretion of immunosuppressive cytokines transforming growth factor- $\beta$ and IL-10 in myeloid cells $(11,46)$.

Blockade of C5aR1 signaling enabled the control of lung metastasis in breast cancer by increasing CD8 T cells and inhibiting regulatory $\mathrm{T}$ cells generation (47).

Thus, it is tempting to speculate that blockade of C5a/C5aR1 may contribute to T-cell effector response in syngeneic models, showing more prominent effects against osseous metastases. Moreover, our study also showed an impact of silenced C5aR1 levels in a model of lung metastasis.
Thus, it is plausible to suggest that besides the skeleton, $\mathrm{C} 5 \mathrm{a} / \mathrm{C} 5 \mathrm{aR} 1$ axis also plays a role in mediating tumor metastasis to other target sites or in conditions of pulmonary cross-seeding form other metastatic organs. A recent report shows that other complement components critically contribute to metastasis to the brain (48). Still, the preliminary finding that primary tumors that metastasize to bone show higher C5aR1 levels than those that metastasize to other organs suggests that this axis might be especially relevant in skeletal metastases.

The therapeutic benefit of C5a/C5aR1 blockade in tumor cells together with its role in cells involved in immune responses provides a solid proof-of-concept for a potential synergism with therapeutic approaches aimed to overcome tumor immune suppression. Antitumor T-cell reactivation is the basis for the current use of therapies blocking the binding of immune checkpoints CTLA-4 and PD-1 to their cognate ligands CD80/86 and $\mathrm{PD}-\mathrm{L} 1 / 2$, respectively (49). In this regard, combined blockade of $\mathrm{PD}-1$ plus $\mathrm{C} 3 \mathrm{aR}$ and $\mathrm{C} 5 \mathrm{aR} 1$ antagonists further enhanced the antitumor effects of $\mathrm{C} 3 \mathrm{aR}$ and $\mathrm{C} 5 \mathrm{aR} 1$ inhibition, an effect attributed to the IL-10mediated recruitment of CD8 T-infiltrating lymphocytes (12). More recently, using different preclinical models of lung cancer growth and metastasis, we have demonstrated the synergistic therapeutic effect of the combined blockade of PD-1 and C5a, using the L-aptamer AON-D21 (33). 
A

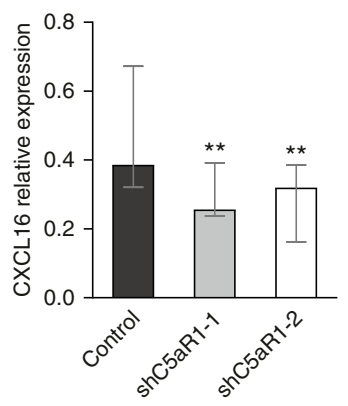

C

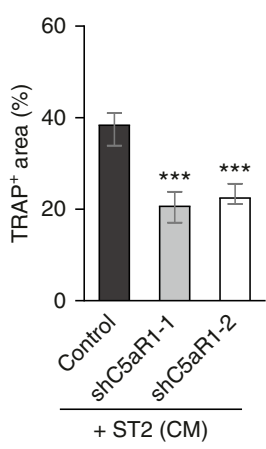

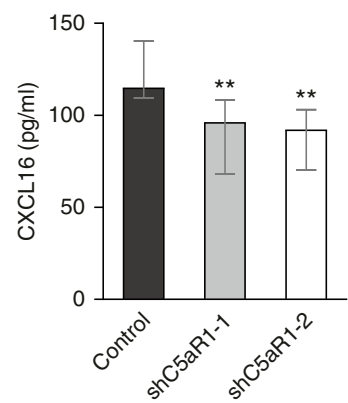

D
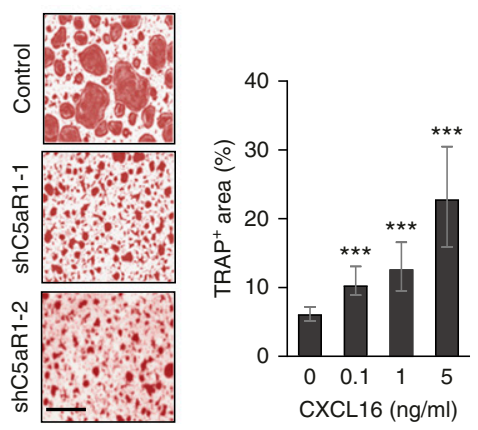

B
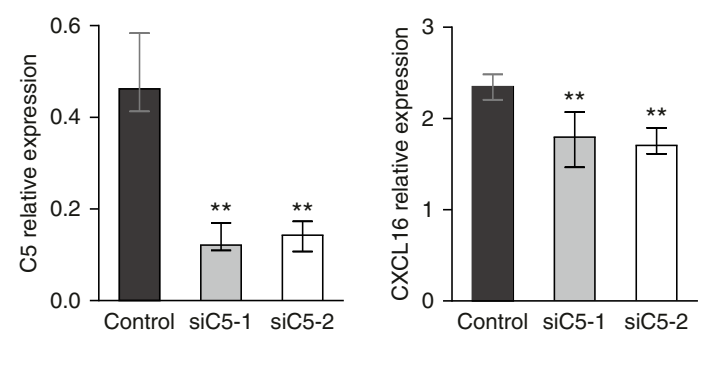

$\mathbf{F}$
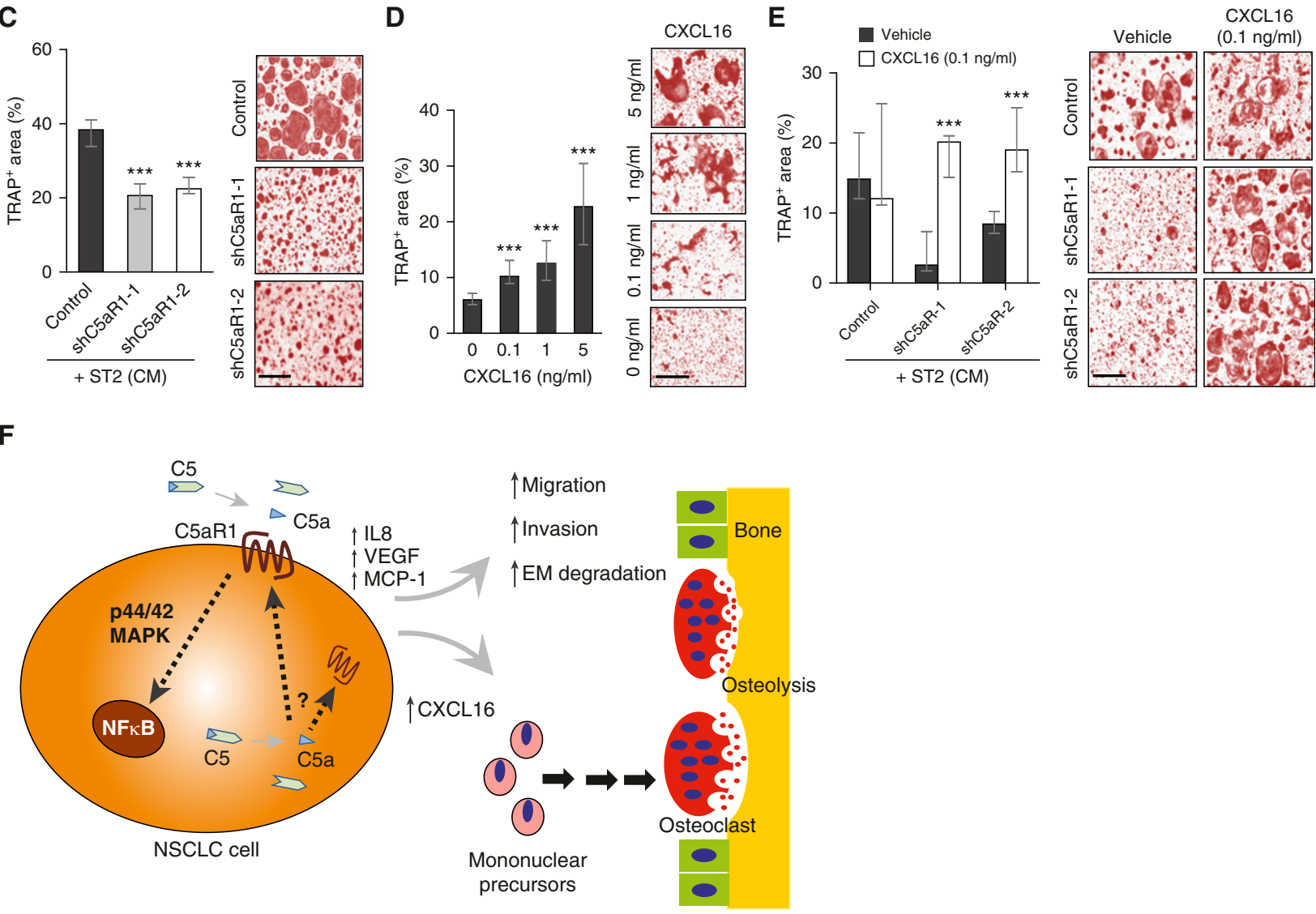

Figure 6. Knockdown of C5aR1 or C5 decreases CXCL16 and osteoclastogenesis. (A) CXCL16 mRNA levels were assessed in parental and knockdown (shC5aR1-1 and -2) A549M1 cells by real-time PCR (left), and secreted levels of CXCL16 were quantified by ELISA in the supernatant from serum-free cultures after 24 hours (right). (B) C5 and CXCL16 expression levels were assessed by real-time PCR in A549M1 cells after transfection with two independent C5-siRNAs (siC5-1 and -2). (C) Left: Conditioned media derived from A549M1 cells transduced with control or shC5aR1 cocultured with ST2 cells were collected and incubated with murine bone marrow mononuclear cells for 6 days in the presence of macrophage colony-stimulating factor (20 ng/ml) and RANKL (15 ng/ml). This experiment was performed four times with similar results. Right: Representative images of osteoclasts (scale bar, $200 \mu \mathrm{m}$; applies to all). (D) Murine bone marrow mononuclear cells were incubated for 6 days in the presence of macrophage colony-stimulating factor (20 ng/ml), RANKL (15 ng/ml), and different doses of CXCL16. Right: Representative images of osteoclasts (scale bar, $200 \mu \mathrm{m}$; applies to all). This experiment was performed twice with similar results. (E) Conditioned medium derived from A549M1 cells transduced with control or shC5aR1 cocultured with ST2 cells were collected, supplemented, or not with $100 \mathrm{pg} / \mathrm{ml}$ of recombinant CXCL16, and incubated with murine bone marrow mononuclear cells for 6 days in the presence of macrophage colony-stimulating factor (20 ng/ml) and RANKL (15 ng/ml). Right: Representative images of osteoclasts (scale bar, $200 \mu \mathrm{m}$; applies to all). (F) Schematic model summarizing that C5a activation from C5 binds to C5aR1 in non-small cell lung cancer cells and triggers p42/44 MAPK phosphorylation and nuclear factor-кB translocation. Intracellular C5a also contributes to C5aR1 signaling. This C5a/C5aR1 axis triggers the secretion of osteoclastogenic factors, which promote bone metastasis by enhancing tumor cell migration, invasion, bone matrix degradation, and CXCL16-mediated osteoclastogenesis. Data are presented as medians and interquartile ranges. ${ }^{\star \star} P<0.01 ;{ }^{* \star \star} P<0.001$. C5a $=$ cleaved complement component of C5; C5aR1 = receptor 1 for C5a; CM = conditioned media; CXCL16 = chemokine (C-X-C) motif ligand 16; EM= extracellular matrix; MAPK = mitogen-activated protein kinase; $\mathrm{MCP}=$ monocyte chemotactic protein; NF-кB = nuclear factor-кB; NSCLC = non-small cell lung cancer; RANKL = receptor activator of nuclear factor $\kappa \mathrm{B}$ ligand; shC5aR1-1 = short hairpin-mediated silencing of C5aR1 gene expression, using shRNA-1; shC5aR1-2 = short hairpin-mediated silencing of C5aR1 gene expression, using shRNA-2; TRAP = tartrate-resistant acid phosphatase; VEGF = vascular endothelial growth factor. 
In relation to the source of $\mathrm{C} 5 \mathrm{a}$ in the tumor microenvironment, complement activation has been described in many different tumor types (1). Interestingly, lung cancer cells in culture are able to produce detectable levels of C5a, and this anaphylatoxin is increased in blood from patients with lung cancer (4). In breast tumor-bearing mice, activation of C5aR1 signaling has been observed with an enhanced synthesis of C3 and C5 in the liver, leading to increased levels of circulating C5a (47). One could speculate that the production of $\mathrm{C} 5 \mathrm{a}$ could be stimulated by soluble factors released by tumor-stromal interactions during osseous colonization. During tumorigenesis and metastasis, $\mathrm{C} 5 \mathrm{aR} 1$ levels might also be influenced by proinflammatory signals from the tumor milieu, or may change during the course of evolution of established lesions, including the impact of therapeutic regimens, such as chemotherapy and/or radiotherapy, an issue that should be properly addressed in future experiments. In addition, our findings also unveil another concomitant mechanism that involves intracellular C5. This could explain why inhibition of extracellular C5a by the aptamer was not as robust as abrogation of $\mathrm{C} 5 \mathrm{aR} 1$, opening new avenues for therapeutic discovery.

In summary, our results demonstrate the mechanistic involvement of the $\mathrm{C} 5 \mathrm{a} / \mathrm{C} 5 \mathrm{aR} 1$ axis in osseous colonization of lung cancer cells. Blocking this ligand/receptor interaction impairs bone metastatic progression, representing a novel target for an effective treatment of lung cancer bone metastases. Our findings add on the emerging role of complement components in lung tumor progression and metastasis and offer a new option in the increasing number of combinatorial regimens that might offer greater efficacy $(50,51)$. Further studies may develop this promising field for translational research and clinical intervention.

\section{Author disclosures are available with the text} of this article at www.atsjournals.org.

\begin{abstract}
Acknowledgment: The authors thank Susana Martínez-Canarias, Cristina Sainz, Ana Remírez, and Amaya Lavín for technical assistance. They are indebted to the members of the Morphology and Image Core facility, especially to Laura Guembe and David Corbacho, and all members of the Animal Core Facility at Center for Applied Medical Research. They also thank Axel Vater and Kai Hoehlig for providing AON-D21 and revAON-D21 (from Aptarion biotech) and for the critical review of the manuscript.
\end{abstract}

\section{References}

1. Pio R, Ajona D, Lambris JD. Complement inhibition in cancer therapy. Semin Immunol 2013;25:54-64.

2. Klos A, Tenner AJ, Johswich KO, Ager RR, Reis ES, Köhl J. The role of the anaphylatoxins in health and disease. Mol Immunol 2009;46: 2753-2766.

3. Croker DE, Monk PN, Halai R, Kaeslin G, Schofield Z, Wu MC, et al. Discovery of functionally selective C5aR2 ligands: novel modulators of C5a signalling. Immunol Cell Biol 2016;94:787-795.

4. Corrales L, Ajona D, Rafail S, Lasarte JJ, Riezu-Boj JI, Lambris JD, et al. Anaphylatoxin $\mathrm{C} 5$ a creates a favorable microenvironment for lung cancer progression. J Immunol 2012;189:4674-4683.

5. Ajona D, Castaño Z, Garayoa M, Zudaire E, Pajares MJ, Martinez A, et al. Expression of complement factor $\mathrm{H}$ by lung cancer cells: effects on the activation of the alternative pathway of complement. Cancer Res 2004;64:6310-6318.

6. Ajona D, Pajares MJ, Corrales L, Perez-Gracia JL, Agorreta J, Lozano MD, et al. Investigation of complement activation product $\mathrm{c} 4 \mathrm{~d}$ as a diagnostic and prognostic biomarker for lung cancer. $J$ Natl Cancer Inst 2013;105:1385-1393.

7. Gu J, Ding JY, Lu CL, Lin ZW, Chu YW, Zhao GY, et al. Overexpression of CD88 predicts poor prognosis in non-small-cell lung cancer. Lung Cancer 2013;81:259-265.

8. Ajona D, Hsu YF, Corrales L, Montuenga LM, Pio R. Down-regulation of human complement factor $\mathrm{H}$ sensitizes non-small cell lung cancer cells to complement attack and reduces in vivo tumor growth. J Immunol 2007;178:5991-5998.

9. Okroj M, Hsu YF, Ajona D, Pio R, Blom AM. Non-small cell lung cancer cells produce a functional set of complement factor I and its soluble cofactors. Mol Immunol 2008;45:169-179.

10. Markiewski MM, DeAngelis RA, Benencia F, Ricklin-Lichtsteiner SK, Koutoulaki A, Gerard C, et al. Modulation of the antitumor immune response by complement. Nat Immunol 2008;9:1225-1235.

11. Gunn L, Ding C, Liu M, Ma Y, Qi C, Cai Y, et al. Opposing roles for complement component $\mathrm{C} 5 \mathrm{a}$ in tumor progression and the tumor microenvironment. J Immunol 2012;189:2985-2994.

12. Wang Y, Sun SN, Liu Q, Yu YY, Guo J, Wang K, et al. Autocrine complement inhibits IL10-dependent T-cell-mediated antitumor immunity to promote tumor progression. Cancer Discov 2016;6: 1022-1035.

13. Nunez-Cruz S, Gimotty PA, Guerra MW, Connolly DC, Wu YQ, DeAngelis RA, et al. Genetic and pharmacologic inhibition of complement impairs endothelial cell function and ablates ovarian cancer neovascularization. Neoplasia 2012;14:994-1004.

14. Imamura T, Yamamoto-Ibusuki M, Sueta A, Kubo T, Irie A, Kikuchi K, et al. Influence of the C5a-C5a receptor system on breast cancer progression and patient prognosis. Breast Cancer 2016;23:876-885.

15. Cho MS, Vasquez HG, Rupaimoole R, Pradeep S, Wu S, Zand B, et al Autocrine effects of tumor-derived complement. Cell Reports 2014; 6:1085-1095.

16. Wada Y, Maeda Y, Kubo T, Kikuchi K, Eto M, Imamura T. C5a receptor expression is associated with poor prognosis in urothelial cell carcinoma patients treated with radical cystectomy or nephroureterectomy. Oncol Lett 2016;12:3995-4000.

17. Nitta H, Wada Y, Kawano Y, Murakami Y, Irie A, Taniguchi K, et al. Enhancement of human cancer cell motility and invasiveness by anaphylatoxin C5a via aberrantly expressed C5a receptor (CD88). Clin Cancer Res 2013;19:2004-2013.

18. Maeda Y, Kawano Y, Wada Y, Yatsuda J, Motoshima T, Murakami Y, et al. C5aR is frequently expressed in metastatic renal cell carcinoma and plays a crucial role in cell invasion via the ERK and PI3 kinase pathways. Oncol Rep 2015;33:1844-1850.

19. Hu WH, Hu Z, Shen X, Dong LY, Zhou WZ, Yu XX. C5a receptor enhances hepatocellular carcinoma cell invasiveness via activating ERK1/2-mediated epithelial-mesenchymal transition. Exp Mol Pathol 2016;100:101-108.

20. Weilbaecher KN, Guise TA, McCauley LK. Cancer to bone: a fatal attraction. Nat Rev Cancer 2011;11:411-425.

21. Vicent S, Perurena N, Govindan R, Lecanda F. Bone metastases in lung cancer. Potential novel approaches to therapy. Am J Respir Crit Care Med 2015;192:799-809.

22. Hernández I, Moreno JL, Zandueta C, Montuenga L, Lecanda F. Nove alternatively spliced ADAM8 isoforms contribute to the aggressive bone metastatic phenotype of lung cancer. Oncogene 2010;29: 3758-3769.

23. Vicent S, Luis-Ravelo D, Antón I, García-Tuñón I, Borrás-Cuesta F, Dotor $\mathrm{J}$, et al. A novel lung cancer signature mediates metastatic bone colonization by a dual mechanism. Cancer Res 2008;68: 2275-2285.

24. Luis-Ravelo D, Antón I, Zandueta C, Valencia K, Pajares MJ, Agorreta J, et al. RHOB influences lung adenocarcinoma metastasis and resistance in a host-sensitive manner. Mol Oncol 2014;8:196-206.

25. Hsu YF, Ajona D, Corrales L, Lopez-Picazo JM, Gurpide A, Montuenga LM, et al. Complement activation mediates cetuximab inhibition of 
non-small cell lung cancer tumor growth in vivo. Mol Cancer 2010;9: 139.

26. Catena R, Luis-Ravelo D, Antón I, Zandueta C, Salazar-Colocho P, Larzábal L, et al. PDGFR signaling blockade in marrow stroma impairs lung cancer bone metastasis. Cancer Res 2011;71:164-174.

27. Luis-Ravelo D, Antón I, Zandueta C, Valencia K, Ormazábal C, Martínez-Canarias S, et al. A gene signature of bone metastatic colonization sensitizes for tumor-induced osteolysis and predicts survival in lung cancer. Oncogene 2014;33:5090-5099.

28. Ignatius $A$, Schoengraf $P$, Kreja L, Liedert A, Recknagel S, Kandert $S$, et al. Complement C3a and C5a modulate osteoclast formation and inflammatory response of osteoblasts in synergism with IL-1 $\beta$. J Cell Biochem 2011;112:2594-2605.

29. Sawant A, Ponnazhagan S. Myeloid-derived suppressor cells as osteoclast progenitors: a novel target for controlling osteolytic bone metastasis. Cancer Res 2013;73:4606-4610.

30. Danilin S, Merkel AR, Johnson JR, Johnson RW, Edwards JR, Sterling JA. Myeloid-derived suppressor cells expand during breast cancer progression and promote tumor-induced bone destruction. Oncolmmunology 2012;1:1484-1494.

31. Ajona D, Zandueta C, Corrales L, Pajares C, Martinez-Terroba E, De Miguel F, et al. Blockade of C5aR impairs bone metastases by decreasing osteoclastic activity. Presented at the 2016 Annual Meeting of the American Society for Bone and Mineral Research. September 16-19 2016, Atlanta, GA. Abstract 1101, p. S33.

32. Travis WD, Brambilla E, Müller-Hermelink HK, Harris CC. Pathology and genetics. Tumours of the lung, pleura, thymus and heart. Lyon: IARC; 2004

33. Ajona D, Ortiz-Espinosa S, Moreno H, Lozano T, Pajares MJ, Agorreta J, et al. A combined PD-1/C5a blockade synergistically protects against lung cancer growth and metastasis. Cancer Discov 2017;7:694-703.

34. Hoehlig K, Maasch C, Shushakova N, Buchner K, Huber-Lang M, Purschke WG, et al. A novel C5a-neutralizing mirror-image (I-) aptamer prevents organ failure and improves survival in experimental sepsis. Mol Ther 2013;21:2236-2246.

35. Antón I, Molina E, Luis-Ravelo D, Zandueta C, Valencia K, Ormazabal C, et al. Receptor of activated protein $\mathrm{C}$ promotes metastasis and correlates with clinical outcome in lung adenocarcinoma. $\mathrm{Am} \mathrm{J}$ Respir Crit Care Med 2012;186:96-105.

36. Freund A, Jolivel V, Durand S, Kersual N, Chalbos D, Chavey C, et al. Mechanisms underlying differential expression of interleukin-8 in breast cancer cells. Oncogene 2004;23:6105-6114.

37. Sarvaiya PJ, Guo D, Ulasov I, Gabikian P, Lesniak MS. Chemokines in tumor progression and metastasis. Oncotarget 2013;4:2171-2185.
38. Hu W, Liu Y, Zhou W, Si L, Ren L. CXCL16 and CXCR6 are coexpressed in human lung cancer in vivo and mediate the invasion of lung cancer cell lines in vitro. PLoS One 2014;9:e99056.

39. Mir H, Singh R, Kloecker GH, Lillard JW Jr, Singh S. CXCR6 expression in non-small cell lung carcinoma supports metastatic process via modulating metalloproteinases. Oncotarget 2015;6:9985-9998.

40. Hu W, Zhen X, Xiong B, Wang B, Zhang W, Zhou W. CXCR6 is expressed in human prostate cancer in vivo and is involved in the in vitro invasion of PC3 and LNCap cells. Cancer Sci 2008;99: 1362-1369.

41. Ha HK, Lee W, Park HJ, Lee SD, Lee JZ, Chung MK. Clinical significance of CXCL16/CXCR6 expression in patients with prostate cancer. Mol Med Rep 2011;4:419-424.

42. Arbore G, Kemper C, Kolev M. Intracellular complement - the complosome - in immune cell regulation. Mol Immunol 2017;89: 2-9.

43. Morgan AJ, Guillen C, Symon FA, Huynh TT, Berry MA, Entwisle JJ, et al. Expression of CXCR6 and its ligand CXCL16 in the lung in health and disease. Clin Exp Allergy 2005;35:1572-1580.

44. Li CH, Xu LL, Zhao JX, Sun L, Yao ZQ, Deng XL, et al. CXCL16 upregulates RANKL expression in rheumatoid arthritis synovial fibroblasts through the JAK2/STAT3 and p38/MAPK signaling pathway. Inflamm Res 2016;65:193-202.

45. Schoengraf $P$, Lambris JD, Recknagel S, Kreja L, Liedert A, Brenner RE, et al. Does complement play a role in bone development and regeneration? Immunobiology 2013;218:1-9.

46. Janelle V, Lamarre A. Role of the complement system in NK cellmediated antitumor T-cell responses. Oncolmmunology 2014;3: e27897.

47. Vadrevu SK, Chintala NK, Sharma SK, Sharma P, Cleveland C, Riediger L, et al. Complement c5a receptor facilitates cancer metastasis by altering T-cell responses in the metastatic niche. Cancer Res 2014; 74:3454-3465

48. Boire A, Zou Y, Shieh J, Macalinao DG, Pentsova E, Massague J. Complement component 3 adapts the cerebrospinal fluid for leptomeningeal metastasis. Cell 2017;168:1101-1113.

49. Pardoll DM. The blockade of immune checkpoints in cancer immunotherapy. Nat Rev Cancer 2012;12:252-264.

50. Melero I, Berman DM, Aznar MA, Korman AJ, Pérez Gracia JL, Haanen J. Evolving synergistic combinations of targeted immunotherapies to combat cancer. Nat Rev Cancer 2015;15:457-472.

51. Sharma P, Allison JP. Immune checkpoint targeting in cancer therapy: toward combination strategies with curative potential. Cell 2015;161: 205-214. 